\title{
Building 3D Statistical Shape Models of Horticultural Products
}

\author{
Femke Danckaers $^{1, *}$. Toon Huysmans ${ }^{1}$. \\ Mattias Van Dael ${ }^{2}$. Pieter Verboven ${ }^{2}$. \\ Bart Nicolaï ${ }^{2} \cdot$ Jan Sijbers ${ }^{1}$ \\ Received: date / Accepted: date
}

\begin{abstract}
A method to build a 3D statistical shape model of horticultural products is described. The framework consists of two parts. First, the surfaces of the horticultural products, which are extracted from X-ray CT scans, are registered to obtain meaningful correspondences between the surfaces. In the second part, a statistical shape model is built from these corresponded surfaces, which maps out the variability of the surfaces and allows to generate new, realistic surfaces. The proposed shape modelling method is applied to 30 Jonagold apples, 30 bell peppers, and 52 zucchini. The average geometric registration error between the original instance and the deformed reference instance is $0.015 \mathrm{~mm} \pm 0.011 \mathrm{~mm}$

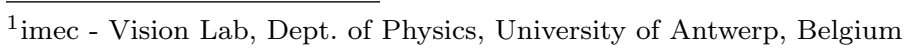
Universiteitsplein 1, Building N, B-2610 Antwerp, Belgium Tel.: +32 (3) 2652477

E-mail: femke.danckaers@uantwerpen.be

${ }^{2}$ Division of Mechatronics, Biostatistics and Sensors (MeBioS), Dept. of Biosystems, K.U.Leuven, Belgium

Kasteelpark Arenberg 30, B-3001 Heverlee, Belgium
\end{abstract}


for the apple dataset, $0.106 \mathrm{~mm} \pm 0.026 \mathrm{~mm}$ for the bell pepper dataset, and $0.027 \mathrm{~mm} \pm 0.007 \mathrm{~mm}$ for the Zucchini dataset. All shape models are shown to be an excellent representation of their specific population, as they are compact and able to generalize to an unseen sample of the population.

Keywords statistical shape model $\cdot$ parameterization $\cdot$ apple $\cdot$ zucchini $\cdot$ bell pepper

\section{Introduction}

Capturing the variability in geometry of vegetables and fruit is important in many aspects of food processing, such as object detection and shape prediction (Costa et al, 2011). Rakun et al (2012) used digital camera images to detect fruit for automatically picking fruit using robots. Prominent shapes were detected by Canny edge detection and Hough transformation. With a statistical shape model, fruit could be detected from one single view, while simultaneously estimating the 3D shape. (Peng and Lu, 2006) predicted the firmness of apple fruit from multispectral scattering images. Light scattering profiles are influenced by the shape of the apple. With a statistical shape model, the shape of the apple could be estimated from limited views to reduce the shape influence, to improve the firmness prediction. Barnea et al (2016) used RGB-D images to detect fruit hanging on a tree. They combined color with the 3D surface normal features, 3D plane-reflective symmetry, and image plane highlights from elliptic surface points to provide shape-based detection of fruits in 3D space regardless of their color. Their method, however, assumes fruit to be symmetric. 
In Zadravec et al (2013), a regression analysis was used to predict the final size of fruit based on the diameter at different growth stages. Statistical shape models could be used to incorporate a more complex description of the shape in the regression, likely improving the prediction results. Moreover, statistical shape models could be valuable to estimate the volume of a fruit from one single view (Iqbal et al, 2011), or to search for a correlation between the stages of growth (Stajnko et al, 2013). A challenging problem is the estimation of the 3D shape to build high resolution dose maps for radiation treatment of heterogeneous food products (Borsa et al, 2002; Kim et al, 2007), as it is important to determine the required irradiation dose. Statistical shape models could lead to a more accurate description of the 3D shape, which would in turn allow to better predict dose deposits. Geometry modeling of food is important for the food engineering domain (Goni et al, 2007, 2008). Realistic 3D models of Conference pears have served to develop nondestructive methods for measuring fruit firmness (Jancsok et al, 2001). Fruit storage designers may use fruit shape models to evaluate the effect of the shape of the fruit on airflow characteristics and thus cooling uniformity (Dehghannya et al, 2010; Muhammad, 2015; Scheerlinck et al, 2004; Verboven et al, 2006; Ho et al, 2011), or to solve stacking problems (Delele et al, 2008).

Statistical shape models are widely used in medical imaging research in a very similar way for modeling organs like the brain (Crum et al, 2016), the heart (Bruse et al, 2016), to model bones like the incudomallear complex (Soons et al, 2016) or the femur (Blanc et al, 2012; Dijck et al, 2014). Shape modeling, however, is relatively novel in food science.

To build a shape model, the correspondences between the surfaces in the object class have to be determined. One option is to annotate the corresponding points 
manually, but this is time-consuming and error prone. A more feasible option is surface registration. With this technique, corresponding points are automatically found by registering each object with the same template surface.

Current methods to build shape models of horticultural products are either based on 2D contour models (Sayinci et al, 2015; Moreda et al, 2012), whereas only a limited number of views is characterized, or simplified 3D models based on contours (Rogge et al, 2015; Mebatsion et al, 2011). The disadvantage of this technique is that the objects have to be star-shaped, so each point of the surface must be reachable with a straight line from a common center without intersecting the surface. In the work of Torppa et al (2007), the shape of potato tubers is approached with ellipsoids, combined with spherical harmonics. However, the use of simple 3D geometrical shapes, such as a cylinders, spheres or ellipsoids, may be too restrictive to describe the complexity of object shapes.

Tornincasa et al (2016) and Ling et al (2007) approached the shape of a hazelnut by starting from a conceptual model, which was modified based on some measured features. With our suggested approach, the entire shape of the object is characterized. Therefore, our technique is applicable to more complex shapes, which may lead to better and more accurate decisions in the applications.

The main goal of our work is to introduce a surface registration framework that provides an accurate geometric fit while maintaining the correspondences and apply it to capture the shape variability of horticultural products (Danckaers et al, 2014). Correspondences between the surfaces are obtained by elastic surface registration. An initial alignment step is added to improve correspondence compared to Amberg et al (2007). With these correspondences, a model can be generated that is compact, (i.e. has few parameters), is highly specific, (i.e. only describes 
horticultural products of a certain class), but also with sufficient generalization ability to be able to describe new instances of that class.

The first part of the framework is $3 \mathrm{D}$ elastic surface registration to obtain correspondences. Amberg et al (2007) presented an algorithm in which each vertex is displaced separately by an affine transformation matrix. They introduced a stiffness parameter in the registration procedure, causing a vertex to be displaced along with its neighbors. During the iterations, the stiffness value is decreased, allowing a more elastic deformation, which results in a good geometric fit, but often suboptimal correspondences. In our approach, an initializing globally affine step is added. Therefore, using only translation of the vertices is sufficient. Furthermore, no landmarks are needed. Therefore, the overall computation time is reduced and the correspondence quality is improved (Danckaers et al, 2014). In the second part of our framework, a shape model is built from the corresponded surfaces by performing principal components analysis (PCA) on the corresponding points of the population (Cootes et al, 1995). In this model, the mean surface and the main variations are incorporated. The last part is the parameterization of the statistical shape model. Because of the correspondences, any shape that can be formed by the model, can also be fit with CAD primitives and consequently can be used for finite element methods such as CFD, e.g. to simulate airflows in fruit packages.

\section{Materials and Methods}

\subsection{Data Collection}

Datasets of three types of fruit and vegetables were scanned in $3 \mathrm{D}$ to validate our algorithm. In particular, 30 Jonagold apples and 30 bell peppers were scanned us- 
ing micro-CT. To this end, a set of 938 2D radiographic images were equiangularly acquired over 187 degrees and are reconstructed to a 3D tomography. The result is a 3D image that represents the X-ray attenuation properties of the scanned object. In this case, the data was acquired with a microfocus X-ray CT (AEA Tomohawk, Philips, The Netherlands) using a Philips HOMX 161 X-ray source. The resulting images had an isotropic voxel size ranging between 83 to $138 \mu \mathrm{m}$. The outer surface of each instance was extracted from the reconstructed objects.

For the zucchini dataset, 52 instances were scanned with an Artec Eva ${ }^{\mathrm{TM}}$ structured light scanner, with a resolution of the scanner is $0.5 \mathrm{~mm}$ and an accuracy of $0.1 \mathrm{~mm}$. This $3 \mathrm{D}$ scanner is able to acquire $2 M$ points per second and has an angular field of view of $30 \times 21^{\circ}$.

The width, depth and height of the instances were measured. The average dimensions of the cultivars are shown in Table 1. All harvesting dates were within the optimal commercial picking window for each cultivar, as determined by the Flanders Centre of Postharvest Technology (VCBT, Belgium).

\subsection{Methods}

In the following sections, the different steps for building a statistical shape model and describing this model with CAD primitives are explained.

\subsubsection{Alignment Initialization}

Before bringing the surfaces into correspondence, the reference surface is rigidly aligned to the target surface by matching their principal axes. Therefore the optimal rotation matrix and translation vector are calculated by following steps: 
1. The centroids $\mathbf{c}_{S}$ and $\mathbf{c}_{T}$ of the uncentered source $\left(\mathbf{S}^{u}\right)$ and uncentered target $\left(\mathbf{T}^{u}\right)$ surface, respectively, are determined. These centroids are subtracted from each point of the surface to align both datasets to the center, resulting in the centered source matrix $\mathbf{S} \in \mathbb{R}^{3 \times n_{S}}$ and centered target matrix $\mathbf{T} \in \mathbb{R}^{3 \times n_{T}}$, with $n_{S}$ the number of source vertices and $n_{T}$ the number of vertices in the target surface.

$$
\begin{aligned}
& \mathbf{c}_{S}=\frac{1}{n_{S}} \sum_{i=1}^{n_{S}} \mathbf{S}_{*, i}^{u} \\
& \mathbf{c}_{T}=\frac{1}{n_{T}} \sum_{i=1}^{n_{T}} \mathbf{T}_{*, i}^{u}
\end{aligned}
$$

2. The covariance matrices of $\mathbf{S}$ and $\mathbf{T}$ are computed, to obtain the symmetric matrices $\mathbf{C}_{S} \in \mathbb{R}^{3 \times 3}$ and $\mathbf{C}_{T} \in \mathbb{R}^{3 \times 3}$, respectively.

$$
\begin{aligned}
\mathbf{C}_{S} & =\frac{1}{n_{S}-1}\left(\mathbf{S} \cdot \mathbf{S}^{T}\right) \\
\mathbf{C}_{T} & =\frac{1}{n_{T}-1}\left(\mathbf{T} \cdot \mathbf{T}^{T}\right) .
\end{aligned}
$$

3. The singular value decompositions (SVD) of both matrices $\mathbf{C}_{S}$ and $\mathbf{C}_{T}$ are calculated to find the principal axes of each surface. The columns of both $\mathbf{U}_{S}$ and $\mathbf{U}_{T}$ correspond with the eigenvectors of $\mathbf{C}_{S}$ and $\mathbf{C}_{T}$, respectively.

$$
\begin{array}{r}
\mathbf{C}_{S}=\mathbf{U}_{S} \mathbf{W}_{S} \mathbf{U}_{S}^{T} \\
\mathbf{C}_{T}=\mathbf{U}_{T} \mathbf{W}_{T} \mathbf{U}_{T}^{T}
\end{array}
$$

4. The rotation matrix $\mathbf{R}$ is found by

$$
\mathbf{R}=\mathbf{U}_{S} \cdot \mathbf{U}_{T}^{T}
$$

and applied to the vertices of the source surface $\mathbf{S}$.

5. The source and target vertices $\mathbf{S}$ and $\mathbf{T}$ are translated by $\mathbf{c}_{S}$.

The alignment algorithm is schematically visualized in Fig. 1. 
2.2.2 Surface Correspondence

In the surface registration part, the reference surface is registered to a target surface, such that the geometric distance between those surfaces becomes minimal while retaining optimal point correspondences. The approach is the same as the RN-ICP-T algorithm, which is also described in previous work of the authors (Danckaers et al, 2014). In the first stage, a closest point correspondence is presumed. Throughout the iterations, the point correspondences gradually improve because of the improved geometric fit. The reference surface is uniformly resampled by the Poisson-disk sampling algorithm (Corsini et al, 2012; Cignoni et al, 2008). A global rigid registration and an elasticity modulated registration are iteratively repeated. During the iterations, the stiffness gradually decreases, allowing the surface to become more elastic throughout the iterations to assure a robust registration algorithm. Hence, in the first iterations a rough alignment is performed to avoid getting stuck in a local minimum, while in the last iterations, the surface will be highly elastic to ensure a perfect geometric fit. The framework is illustrated in Fig. 2.

In the first step of the iterative process, the surfaces are rigidly aligned. To that end, corresponding points are found using normal-ray casting from each vertex of the reference surface to the target surface. The intersection point, that lies on the target surface and is not necessarily a vertex, may be a corresponding point. A number of constraints are imposed on the corresponding points:

1. The normal of the intersection has to point in the same direction (within a tolerance of $30^{\circ}$ ) as the normal of the source point. 
2. The distance between corresponding points has to be smaller than $20 \times$ the average distance between the two surfaces as measured from the previous iteration. In the first iteration, the previous average distance is set to infinity.

3. The casted ray may not intersect the source and target surface multiple times before reaching the corresponding point.

If no corresponding point is found, it has no influence on the alignment of the surfaces. Based on the corresponding points, a linear least squares alignment is performed to determine the transformation matrix that minimizes the distance between the corresponding points.

In the elastic part of the registration the vertices are allowed to move separately, while motion is restricted by a stiffness parameter $\beta$ that regulates the strength of the connection with the neighboring vertices and which gradually decreases during the iterations. Hence, the movement of neighboring vertices is constrained, resulting in similar movements of neighboring vertices, as displayed in Fig. 3. By applying weights to each vertex the influence of this vertex can be set. If no corresponding point for a vertex of the source mesh is found, its weight is set to zero. In that case, this vertex simply moves along with its neighboring vertices. Let $n$ be the number of vertices of the surface and $e$ the number of edges.

The weights corresponding to the vertices are stored as elements of the diagonal matrix $\mathbf{W} \in \mathbb{R}^{n \times n}$, which are either 0 or 1 . In this algorithm, the weight can be either 0 or 1. Matrix $\mathbf{S} \in \mathbb{R}^{n \times 3}$ and matrix $\mathbf{T} \in \mathbb{R}^{n \times 3}$ hold the coordinates of the corresponding source and target vertices, respectively. The optimal translation 
vectors, the matrix $\mathbf{X}_{t} \in \mathbb{R}^{n \times 3}$, are found by solving following linear system:

$$
\left[\begin{array}{l}
\beta \mathbf{M} \\
\mathbf{W I}
\end{array}\right] \mathbf{X}_{t}=\left[\begin{array}{c}
\mathbf{0} \\
\mathbf{W}(\mathbf{T}-\mathbf{S})
\end{array}\right],
$$

with $\mathbf{M} \in \mathbb{R}^{e \times n}$ the incidence matrix of the reference surface that indicates the start- and end vertex of each edge. The rigid registration step and elastic registration steps are iteratively repeated until convergence is reached, which is calculated by comparing the current distance between the source and reference surface $d_{t}$ and the previous distance between the source and reference surface $d_{t-1}$. Convergence is reached if

$$
\frac{\left|d_{t}-d_{t-1}\right|}{d_{t}}<0.001
$$

\subsubsection{Building a Shape Model}

This part of our framework consists of building a statistical shape model based on the corresponded surfaces that resulted from the surface registration part. The process is shown in Fig. 4. To build a shape model, it is important that the surfaces are superimposed by optimally translating and rotating the surfaces. The optimal poses are determined by Procrustes alignment (Dryden and Mardia, 1998; Gower, 1975; Kendall, 1989).

The model is built by performing principal components analysis (PCA) on the matrix containing the corresponding points to compute the eigenvectors and eigenvalues of the covariance matrix across all training shapes. This corresponding 
points matrix $\mathbf{X} \in \mathbb{R}^{N \times 3 n}$ is given by

$$
\mathbf{X}=\left[\begin{array}{ccccccc}
x_{0,0} & y_{0,0} & z_{0,0} & \ldots & x_{0, n} & y_{0, n} & z_{0, n} \\
x_{1,0} & y_{1,0} & z_{1,0} & \ldots & x_{1, n} & y_{1, n} & z_{1, n} \\
& \vdots & \vdots & \vdots & \\
x_{N, 0} & y_{N, 0} & z_{N, 0} & \ldots & x_{N, n} & y_{N, n} & z_{N, n}
\end{array}\right]
$$

with $n$ the number of points and $N$ the number of shapes. The $i$-th row of this corresponding points matrix is denoted by $\mathbf{x}_{i}$.

In the statistical shape model, the mean surface $\overline{\mathbf{x}}$ and the main variances $\boldsymbol{\Phi}$ are incorporated. The population of $N$ shapes is represented by a point cloud with $N$ points in an $3 n$-dimensional space, where each point represents a vegetable or fruit. This cloud can be represented by $N-1$ eigenmode vectors, where the first eigenmode corresponds to the direction of the largest variance in the population, the second eigenmode corresponds to the second direction of the largest variance perpendicular to the first, etc.

The calculation of the statistical shape model is as follows. First, the average shape vector $\overline{\mathbf{x}}$ is calculated by

$$
\overline{\mathbf{x}}=\frac{1}{N} \sum_{i=1}^{N} \mathbf{x}_{i}
$$

The normalized corresponding points matrix $\hat{\mathbf{X}}$ is given by

$$
\hat{\mathbf{X}}=\left[\begin{array}{c}
\mathbf{x}_{0}-\overline{\mathbf{x}} \\
\mathbf{x}_{1}-\overline{\mathbf{x}} \\
\vdots \\
\mathbf{x}_{N}-\overline{\mathbf{x}}
\end{array}\right] .
$$

Then, the normalized $N \times N$ covariance matrix $\mathbf{D}$ is computed as follows:

$$
\mathbf{D}=\frac{1}{N-1} \hat{\mathbf{X}} \hat{\mathbf{X}}^{T}
$$


Next, the eigenvalues $\boldsymbol{\Lambda}$ and eigenvectors $\boldsymbol{\Phi}$ are obtained from the SVD of this covariance matrix $\mathbf{D}$ as

$$
\mathrm{D}=\mathrm{U}_{D} \Lambda_{D} \mathrm{U}_{D}^{T}
$$

where $\boldsymbol{\Lambda} \in \mathbb{R}^{3 n \times 3 n}$ is the diagonal matrix that holds the eigenvalues corresponding to the eigenvectors in the columns of the matrix $\Phi \in \mathbb{R}^{N \times 3 n}$, which is calculated by

$$
\Phi=\hat{\mathbf{X}} \cdot \mathbf{U}_{D}
$$

Any instance $\mathbf{y}$ can be approximated by the average surface $\overline{\mathbf{x}}$ and a weighted sum of principal component parameters $\boldsymbol{\Phi}$ as follows:

$$
\mathrm{y}=\mathrm{C}(\overline{\mathrm{x}}+\Phi \mathrm{b})
$$

where $\mathbf{C}$ is a rigid transformation matrix. The vector $\mathbf{b}$ holds the shape parameters, which are normally distributed with a certain standard deviation, calculated by PCA. This means that a new, realistic surface can be formed by adapting the shape model parameters.

\subsubsection{Description with CAD primitives}

For simulation applications, such as CFD and FEM environments, a shape must be representable with CAD primitives, which are basic geometric shapes such as spheres, cubes, toroids, cylinders, pyramids, and b-splines. These are considered to be primitives in 3D modeling because they are the building blocks for many other shapes and forms. To use a shape model of vegetables or fruit in a CAD environment, shape parameterization is needed.

Parameterization of a surface is the task of defining a map between the surface and a simple parameter domain, such as a plane, sphere, or cylinder. Such a map 
links each point of the surface with a coordinate in the space of the parameter domain. In this paper, the surfaces are represented with a triangle mesh and the map is only defined explicitly for the vertices. Parameterization can be seen as the result of a continuous deformation of the surface into the parameter domain. By parameterizing the shape model, each instance in the model can be easily described with a set of basis functions, like spherical harmonics or B-splines.

The cylindrical parameter domain is chosen in order to be able to select the poles. Therefore, two holes are manually created in the top and bottom of the surface, to be able to work in this domain (Huysmans et al, 2005). A mapping from the cylinder to the triangle mesh of the surface is needed. Therefore the surface mesh is represented by a progressive mesh. With this representation, the number of triangles is reduced until the simplest shape, an open prism with six vertices, is left. This simple shape can be easily parameterized by equidistant placement of its six vertices on the two boundaries of the cylindrical domain. The next levels in the progressive mesh are parameterized by inserting the removed vertices one at a time and optimizing their positions on the cylinder in a way that the mapping between the cylinder and the surface introduces a minimum of distortion. After re-inserting all vertices, the parameterization of the original surface surface is obtained and each vertex has a $(u, v)$ coordinate in the cylindrical coordinate system.

The statistical shape model is parameterized by only parameterizing the average surface. Because of the correspondence, all instances of the model also have B-spline parameter coordinates. With this technique, the point-based models can be described by B-splines, which describe a surface by a set of control points. The 3D locations of the control points are optimized to obtain the best surface 
approximation. This is a very compact representation and is suited for CAD and finite-element environments.

\section{Results and Discussion}

\subsection{Surface Registration}

To obtain meaningful correspondences, a reference surface was registered to each surface of the population. For each class, a reference surface was randomly chosen from the population. The apple was resampled to 30000 points, the bell pepper to 40000 points and the zucchini to 13000 points. The reference surface of each class was registered to each surface of the population. The stiffness parameter decreased linearly from 50 to 1 . Registering one instance took \pm 1 min CPU time for 30 iterations. Then, the average surface was calculated and served as a new template surface to register to each instance to avoid a bias towards a specific shape of the population. In Fig. 5, the deformation of the reference surface to a target surface of the bell pepper object class is visualized through the iterations. From the results of this registration, a statistical shape model was built.

The average error between the source surface and the deformed reference surface for each class is shown in Fig. 6 and summarized in Table 2. The largest errors appeared around the top of the surfaces, where the stem was located. The bell pepper surfaces had the largest errors, because the shape and length of the stem greatly varies over the instances.

The correspondence quality was evaluated for the bell pepper model by manually placing a marker on the top (of the cut off stem) and a marker on the bottom of the input surfaces. Those marker points were also annotated on the average sur- 
face and loaded on each instance incorporated in the model. Next, the Euclidean distance between the manually placed marker and automatically loaded marker was calculated. The results of this analysis are shown in Table 3.

The correspondence error on the bottom of the bell pepper is relatively high. It is difficult to manually annotate this point, because there are no clear features on the bottom of a bell pepper, so the inter- and intra observer errors are large. Moreover, because there are no distinct features on the bottom, even for suboptimal correspondences the similarity criteria, such as the directions of the normals have to be in the same direction within a tolerance of $30^{\circ}$, are easily achieved. Once a bad correspondence is found, it is very unlikely that the algorithm is capable of correcting this.

The geometric and correspondence errors can perhaps be reduced by adding more vertices to the reference surface or lowering the convergence ratio. The more vertices on the reference surface, the better a shape can be captured. However, computation time and memory requirements increase with the number of vertices. Lowering the convergence threshold could possibly lead to better results on the stem and bottom.

\subsection{Shape Model}

In Fig. 7 the first five shape modes of the bell pepper shape model are visualized, since these modes represent over $80 \%$ of the variation. The first mode represents mainly the size of the bell pepper. The second mode shows the ratio between width and height. The third mode describes the location and curvedness of the lobes. 
The characteristics described in the following modes are harder to interpret. They mostly describe the curvature of the bottom of the bell pepper.

It is assumed that a statistical shape model describes a multidimensional Gaussian distribution of the object's shape. As an example, the model was truncated to 5 eigenmodes, because the first components mostly describe the shape. Five samples were randomly generated by generating a weight vector $\mathbf{b}$. These weights were between +3 standard deviations and -3 standard deviations of their respective shape mode and followed the Gaussian distribution. Some randomly generated instances are visualized in Fig. 8.

\subsection{Description with CAD primitives}

Surface parameterization was applied to the average surface of the apple shape model to be able to describe each model instance with basis functions. In Fig. 9, the average apple surface with iso-parametric curves is shown. The apple surface was approximated by B-splines with different numbers of control points. While the size and global shape of an apple can already be described with a $4 \times 4$ grid of control points, the difference between the original and the approximation was clearly visible. An approximation with $32 \times 32$ control points was nearly identical to the original apple model. Therefore the parameterized shape model could serve as a model in CAD and finite-element environments, so simulations could be performed with these models.

Moreover, the parameterized shape model is a much more compact and memory efficient representation of the triangle model. For example, the triangle model of the apple consisted of 30000 points requiring $703 k B$ of storage per surface. On 
the other hand, the most detailed parameterized apple shape consisted of $32 \times 32$ control points only requiring $24 k B$ per surface. So the required memory for the parameterized shape is almost 30 times smaller than for the triangle model. In addition, for the triangle model the vertex connectivity has to be stored while for the B-spline representation the connectivity is implicit.

\subsection{Model Performance}

Compactness, generalization ability and specificity are widely used measures (Davies, 2002; Zihua, 2011) for quantifying the correspondence quality of a statistical shape model. In this section, the different model performance measures were calculated per object type.

\subsubsection{Compactness}

A compact model is a model that can represent all shapes of the class with as little parameters as possible. Preferably, a shape model can be described with few modes. The compactness is expressed as the sum of variances of the model,

$$
C(m)=\sum_{i=1}^{m} \lambda_{i}
$$

where $\lambda_{i}$ is the variance in shape mode $i$, and $C(m)$ is the compactness using $m$ modes.

In Fig. 10, the compactness graph for each object class is shown. The cumulative variance was normalized for each model so that the total was $100 \%$. The compactness test shows that the apple model captured more than $85 \%$ of the shape variation with the first 10 modes. The bell pepper model captured more than $90 \%$ of the shape variation with the first 10 modes. 
Note that the zucchini model is a very compact model, since only 5 modes were needed to describe over $99 \%$ of the total population. This is because the main shape variance is size, which was described by the first mode.

\subsubsection{Generalizability}

Generalizability is defined as the degree to which the model can be generalized from the study sample to the entire population. The shape model should allow to describe all shapes of the specific class, and not only the shapes of the training set. If a model is over-fitted to the training set, it will not be able to generalize to unseen samples.

Generalizability $G(m)$ was measured by performing leave-one-out tests, where a shape model was built by using all training shapes but one. Next, the left-out shape was described by adapting the shape parameters of the model. Generalizability was calculated as the mean error over all left out shapes,

$$
G(m)=\frac{1}{N_{t}} \sum_{i=1}^{N_{t}}\left\|\mathbf{x}_{i}-\mathbf{x}_{i}^{\prime}(m)\right\|^{2},
$$

where $\mathbf{x}_{i}$ is the left-out shape and $\mathbf{x}_{i}^{\prime}(m)$ is the attempted description using the shape model with $m$ modes. The number of trials, or objects in the model, is represented by $N_{t}$.

In Fig. 11, the generalizability graph for each object class is shown. The generalizability error was calculated in $m m$ per vertex. The error of fitting the apple model to an unseen apple surface was smaller than $1.9 \mathrm{~mm}$ from 5 shape modes. For the bell pepper shape model, an error of less than $3.5 \mathrm{~mm}$ occurred when predicting a shape using the first 5 shape modes. The outer surface of a zucchini could be fit with an error of $1.6 \mathrm{~mm}$ from 5 modes. 


\subsubsection{Specificity}

A specific model can only represent instances of the object class that are similar to those in the training set. Specificity was measured by generating an amount of shapes $\left(N_{t}\right)$ by generating a random parameter vector with $m$ modes. Each sample was compared to the most similar shape in the training set. The specificity measure can be expressed as,

$$
S(m)=\frac{1}{N_{t}} \sum_{i=1}^{N_{t}}\left\|\mathbf{y}_{i}-\mathbf{y}_{i}^{\prime}(m)\right\|^{2},
$$

where $\mathbf{y}_{i}^{\prime}$ are shape examples generated by the model and $\mathbf{y}_{i}$ is the nearest member of the training set to $\mathbf{y}_{i}^{\prime}$.

In Fig. 12, the specificity graph for each type of surface is shown. The specificity error was calculated in $\mathrm{mm}$ per vertex. The specificity test proved that all three models were able to generate shapes that resemble those in the training set.

\subsection{Discussion}

The most innovative feature of this algorithm is that it leads to accurate statistical shape models of objects with complex, possibly non-star shaped topologies. The shapes were described in a detailed, realistic way, compared to the current 2D contour models or simplified 3D models based on contours. The algorithm is applicable to vegetables and fruit with a shape more complex than star-shaped (Rogge et al, 2015). Star-shaped means that each point of the surface is reachable with a straight line from a common center without intersecting the surface, which was not the case for some of the bell pepper instances. Therefore, the algorithm can also be applied to e.g. mushrooms and bananas. 
By applying surface registration to a dataset, all instances were in correspondence with each other. The geometric errors and correspondence errors between the deformed reference surface and the target surface were small, so the registration result is a good representation of the input surface. Geometric and correspondence errors could possibly be reduced by changing the stiffness parameters. A small stiffness value leads to a better geometric fit, because the vertices can move more freely to the optimal position. On the other hand, chances are higher that a vertex migrates to an incorrect location because of this freedom. Once a bad correspondence is found and the surface is incorrectly deformed, the algorithm is not capable of correcting this mistake. A large stiffness value leads to a less optimal geometric fit, but the overall shape of the source surface is maintained, increasing the chances of a good correspondence. Other possibilities to reduce the errors are increasing the number of vertices and a more strict convergence criterion.

The algorithm was capable of rapidly generating 3D statistical shape models that were accurate and compact, while the randomly generated surfaces were similar to the surfaces of their respective training sets. Therefore, a broad dataset of realistic instances can be produced for e.g. simulation purposes.

B-spline surface approximation of the shape model was shown. Because the model was parameterized, all instances that can be formed by the model were also parameterized. Therefore, a statistical shape model can provide input to generate CAD models, which can be loaded in CFD and FEM environments. As a result, the shape model is employable in stacking algorithms (Delele et al, 2008). Moreover, the parameterized shape model is a more compact representation of the triangle model, as less numbers have to be stored. 
The software for the described algorithms was written in $\mathrm{C}++$ and based on the Visualization ToolKit (VTK) libraries (Schroeder et al, 2006). VTK is an open source software system for 3D computer graphics, image processing, and visualization.

\section{Conclusions}

In this paper, an algorithm for building a statistical shape model of horticultural products was proposed. First, the surfaces from the training set were brought into correspondence with each other. Experiments on the surface registration algorithm proved that the technique is applicable to complex shapes and results in a good geometric fit and good correspondences. From these corresponded surfaces, the statistical shape model was built. The model performance tests showed that our method for building a statistical shape model results in a good representation of the population of the object class, as the shape model is able to generate realistic horticultural product shapes that differ from those in the training set. Furthermore, the model was a compact representation of the shape population and could easily generalize to a formerly unseen instance of the model's object class.

By parameterizing the surface, the shape model and every instance that can be formed by the shape model, were described by CAD primitives. Therefore, a statistical shape model is an effective tool for simulation software. Our approach of modeling and subsequent parameterization is also applicable to other horticultural product shapes. Specifically for elongated shapes, like pears, bananas, and cucumbers, cylindrical parameterization can be useful. 
The developed surface registration and modeling techniques proposed in this paper are also applicable to other horticultural products of more complicated, non-spherical topology.

Acknowledgements This work was supported by the Agency for Innovation by Science and Technology in Flanders (IWT SB 141520 and IWT SBO 120033 TomFood).

\section{References}

Amberg B, Romdhani S, Vetter T (2007) Optimal step nonrigid ICP algorithms for surface registration. In: Conference on Computer Vision and Pattern Recognition, IEEE, pp 1-8

Barnea E, Mairon R, Ben-Shahar O (2016) Colour-agnostic shape-based 3D fruit detection for crop harvesting robots. Biosystems Engineering

Blanc R, Seiler C, Szkely G, Nolte LP, Reyes M (2012) Statistical model based shape prediction from a combination of direct observations and various surrogates: Application to orthopaedic research. Medical Image Analysis 16(6):1156 $-1166$

Borsa J, Chu R, Sun J, Linton N, Hunter C (2002) Use of CT scans and treatment planning software for validation of the dose component of food irradiation protocols. Radiation Physics and Chemistry 63(3-6):271-275

Bruse JL, McLeod K, Biglino G, Ntsinjana HN, Capelli C, Hsia TY, Sermesant M, Pennec X, Taylor AM, Schievano S (2016) A statistical shape modelling framework to extract $3 \mathrm{~d}$ shape biomarkers from medical imaging data: assessing arch morphology of repaired coarctation of the aorta. BMC Medical Imaging $16(1): 40$ 
Cignoni P, Callieri M, Corsini M, Dellepiane M, Ganovelli F, Ranzuglia G (2008) MeshLab: An open-source mesh processing tool. Eurographics, Italy, pp 129-136

Cootes T, Taylor C, Cooper D, Graham J (1995) Active shape models - their training and application. Computer Vision and Image Understanding 61(1):3859

Corsini M, Cignoni P, Scopigno R (2012) Efficient and flexible sampling with blue noise properties of triangular meshes. IEEE Transactions on Visualization and Computer Graphics 18(6):914-924

Costa C, Antonucci F, Pallottino F, Aguzzi J, Sun DW, Menesatti P (2011) Shape analysis of agricultural products: A review of recent research advances and potential application to computer vision. Food and Bioprocess Technology 4(5):673-692

Crum WR, Danckaers F, Huysmans T, Cotel MC, Natesan S, Modo MM, Sijbers J, Williams SCR, Kapur S, Vernon AC, et al (2016) Chronic exposure to haloperidol and olanzapine leads to common and divergent shape changes in the rat hippocampus in the absence of grey-matter volume loss. Psychological Medicine 46(15):30813093

Danckaers F, Huysmans T, Lacko D, Ledda A, Verwulgen S, Van Dongen S, Sijbers J (2014) Correspondence preserving elastic surface registration with shape model prior. In: ICPR'14, IEEE, pp 2143-2148

Davies RH (2002) Learning shape: Optimal models for analysing natural variability. PhD thesis, University of Manchester

Dehghannya J, Ngadi M, Vigneault C (2010) Mathematical modeling procedures for airflow, heat and mass transfer during forced convection cooling of produce: A review. Food Engineering Reviews 2(4):227-243 
Delele M, Tijskens E, Atalay Y, Ho Q, Ramon H, Nicolaï B, Verboven P (2008) Combined discrete element and CFD modelling of airflow through random stacking of horticultural products in vented boxes. Journal of Food Engineering $89(1): 33-41$

Dijck CV, Wirix-Speetjens R, Huysmans T, Danckaers F, Sijbers J, Vander Sloten J (2014) Influence of correspondence method on statistical model based shape prediction. In: IEEE International Symposium on Biomedical Imaging (ISBI)

Dryden IL, Mardia KV (1998) Statistical shape analysis. Wiley Series in Probability and Statistics, John Wiley \& Sons, Chichester ; New York

Goni SM, Purlis E, Salvadori VO (2007) Three-dimensional reconstruction of irregular foodstuffs. Journal of Food Engineering 82(4):536-547

Goni SM, Purlis E, Salvadori VO (2008) Geometry modelling of food materials from magnetic resonance imaging. Journal of Food Engineering 88(4):561-567

Gower JC (1975) Generalized Procrustes analysis. Psychometrika 40(1):33-51

Ho QT, Verboven P, Verlinden BE, Herremans E, Wevers M, Carmeliet J, Nicolaï BM (2011) A three-dimensional multiscale model for gas exchange in fruit. Plant Physiology 155(3):1158-1168

Huysmans T, Sijbers J, Verdonk B (2005) Parameterization of tubular surfaces on the cylinder. Journal of the Winter School of Computer Graphics 10(3):97-104 Iqbal SM, Gopal A, Sarma ASV (2011) Volume estimation of apple fruits using image processing. In: International Conference on Image Information Processing (ICIIP), IEEE, pp 1-6

Jancsok PT, Clijmans L, Nicolaï BM, De Baerdemaeker J (2001) Investigation of the effect of shape on the acoustic response of 'conference' pears by finite element modelling. Postharvest Biology and Technology 23(1):1-12 
Kendall DG (1989) A survey of the statistical theory of shape. Statistical Science $4(2): 87-99$

Kim J, Moreira R, Huang Y, Castell-Perez M (2007) 3-D dose distributions for optimum radiation treatment planning of complex foods. Journal of Food Engineering 79(1):312-321

Ling L, Hongzhen X, Wenlin S, Gelin L (2007) Research on visualisation of fruits based on deformation. New Zealand Journal of Agricultural Research 50(5):593600

Mebatsion H, Boudon F, Godin C, Pradal C, Génard M, Goz-Bac C, Bertin N (2011) A novel profile based model for virtual representation of quasi-symmetric plant organs. Computers and Electronics in Agriculture 75(1):113-124

Moreda G, Muñoz M, Ruiz-Altisent M, Perdigones A (2012) Shape determination of horticultural produce using two-dimensional computer vision - A review. Journal of Food Engineering 108(2):245-261

Muhammad G (2015) Date fruits classification using texture descriptors and shape-size features. Engineering Applications of Artificial Intelligence 37:361367

Peng Y, Lu R (2006) Improving apple fruit firmness predictions by effective correction of multispectral scattering images. Postharvest Biology and Technology $41(3): 266-274$

Rakun J, Stajnko D, Berk P, Lakota M, Zazula D (2012) Detecting natural objects by means of 2D and 3D shape analysis. In: Actual Tasks on Agricultural Engineering, Croatia, pp 345-354

Rogge S, Defraeye T, Herremans E, Verboven P, Nicolaï BM (2015) A 3D contour based geometrical model generator for complex-shaped horticultural products. 
Journal of Food Engineering 157:24-32

Sayinci B, Kara M, Ercişli S, Duyar Ö, Ertürk Y (2015) Elliptic Fourier analysis for shape distinction of Turkish hazelnut cultivars. Erwerbs-Obstbau 57(1):1-11

Scheerlinck N, Marquenie D, Jancsók PT, Verboven P, Moles CG, Banga JR, Nicolaï BM (2004) A model-based approach to develop periodic thermal treatments for surface decontamination of strawberries. Postharvest Biology and Technology 34(1):39-52

Schroeder W, Martin K, Lorensen B, Kitware I (2006) The visualization toolkit: an object-oriented approach to 3D graphics. Kitware, [Clifton Park, N.Y.]

Soons JA, Danckaers F, Keustermans W, Huysmans T, Sijbers J, Casselman JW, Dirckx JJ (2016) 3d morphometric analysis of the human incudomallear complex using clinical cone-beam ct. Hearing research 340:79-88

Stajnko D, Rozman Č, Pavlovič M, Beber M, Zadravec P (2013) Modeling of Gala apple fruits diameter for improving the accuracy of early yield prediction. Scientia Horticulturae 160:306-312

Tornincasa S, Bonisoli E, Brino M (2016) Parametric, asymmetric and stochasticbased 3D CAD model of Tonda Gentile Trilobata hazelnut variety. Biosystems Engineering 144:72-84

Torppa J, Valkonen JPT, Muinonen K (2007) Three-dimensional stochastic shape modelling for potato tubers. Potato Research 49(2):109-118

Verboven P, Flick D, Nicolaï B, Alvarez G (2006) Modelling transport phenomena in refrigerated food bulks, packages and stacks: basics and advances. International Journal of Refrigeration 29(6):985-997

Zadravec P, Veberic R, Stampar F, Eler K, Schmitzer V (2013) Fruit size prediction of four apple cultivars: Accuracy and timing. Scientia Horticulturae 160:177-181 
Zihua S (2011) Statistical shape modelling: Automatic shape model building. PhD thesis, University College London 

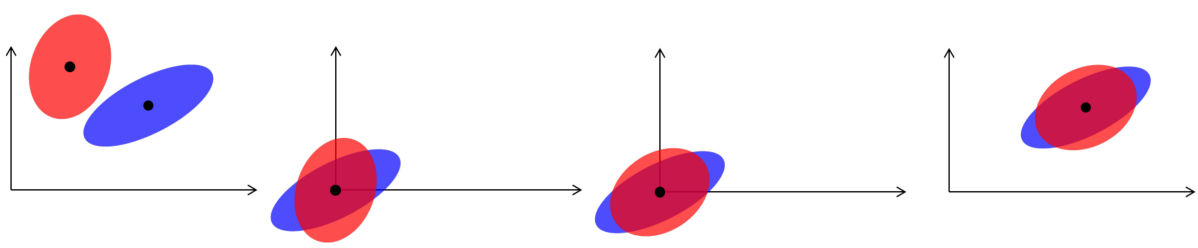

Fig. 1: Schematic 2D visualization of the alignment of two surfaces. The red and blue ellipse represent the source and target surface, respectively. First, the centroids of both surfaces are calculated. Next, the surfaces are aligned to the origin. The following step is rotation of the source surface such that its principal axes align with those of the target surface. Finally, both surfaces are translated to the optimal location by the centroid of the source surface.

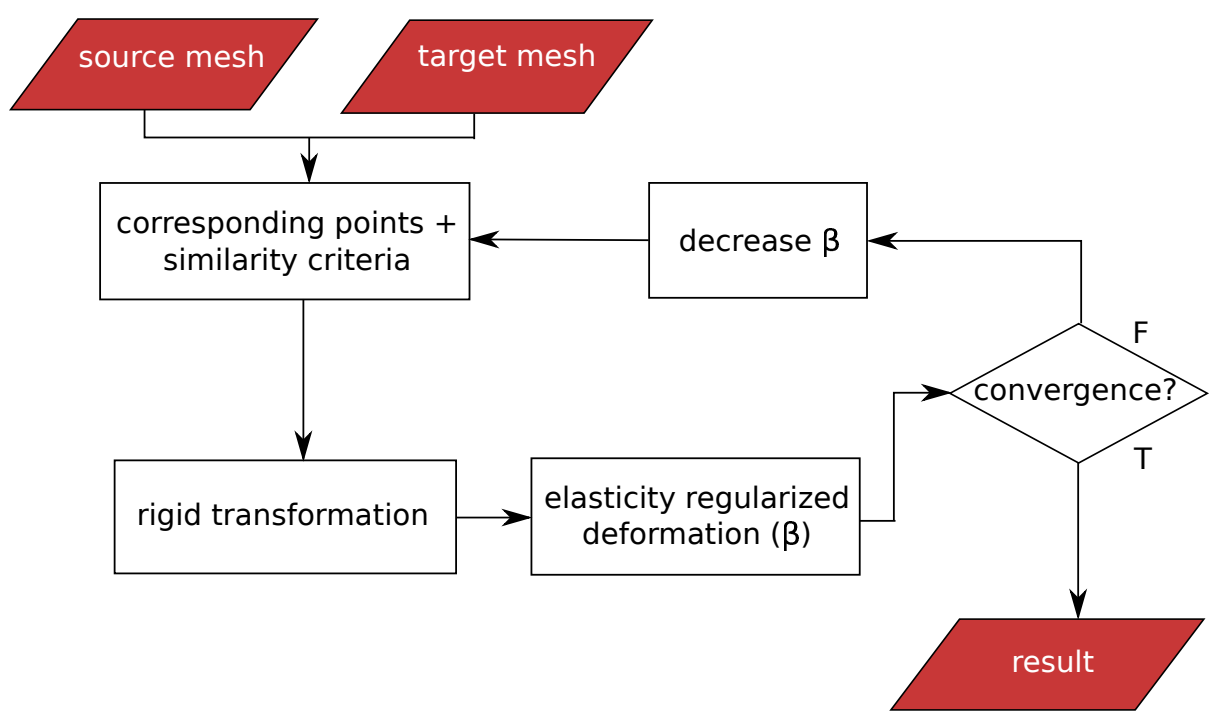

Fig. 2: The surface registration framework. 

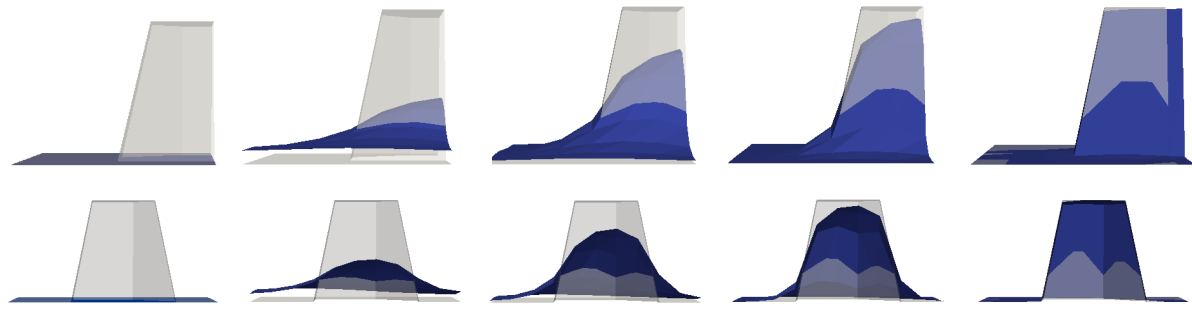

$\beta / 10$
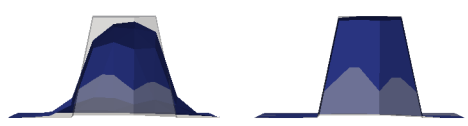

$\beta / 20$

$\beta / 100$

Fig. 3: Influence of the elasticity parameter on the elastic surface deformation, shown from two viewpoints. In each figure, the target surface is visualized in transparent white and the deformed source in blue. The left example is created with a very high stiffness factor. The right example is created with a very low stiffness factor. From left to right, the stiffness factor decreases.

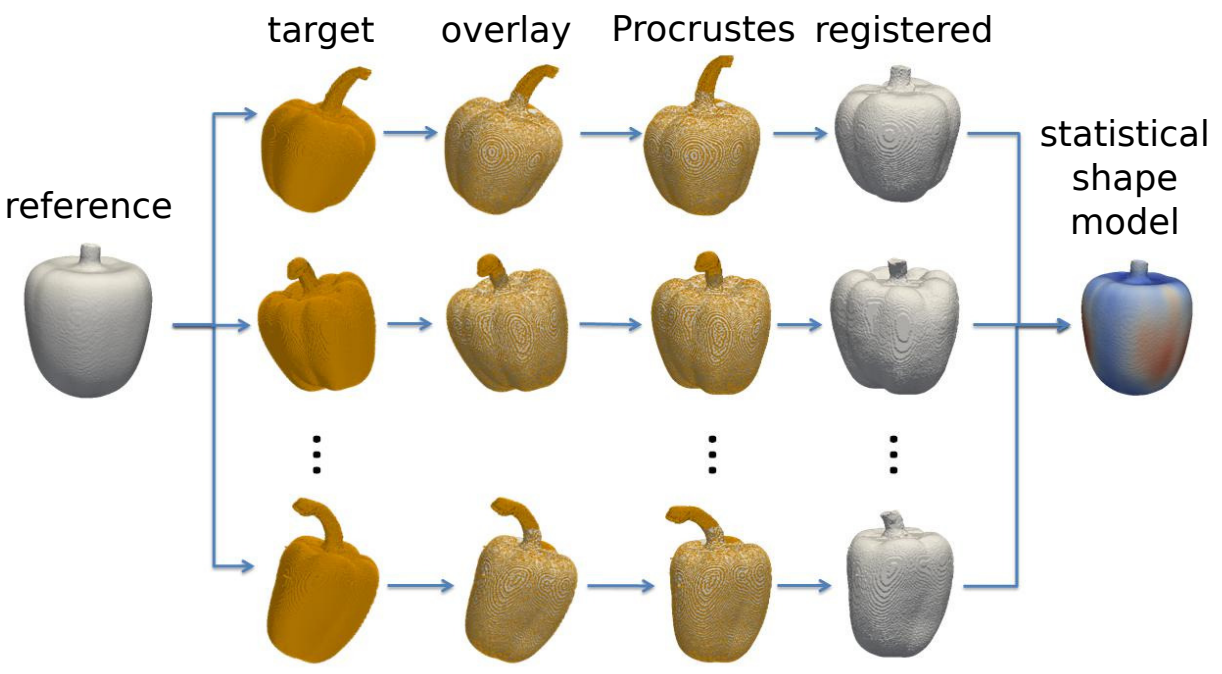

Fig. 4: Framework for building a statistical shape model. First, a reference surface is registered to each surface of the population. From these registered surfaces, a statistical shape model is built. 


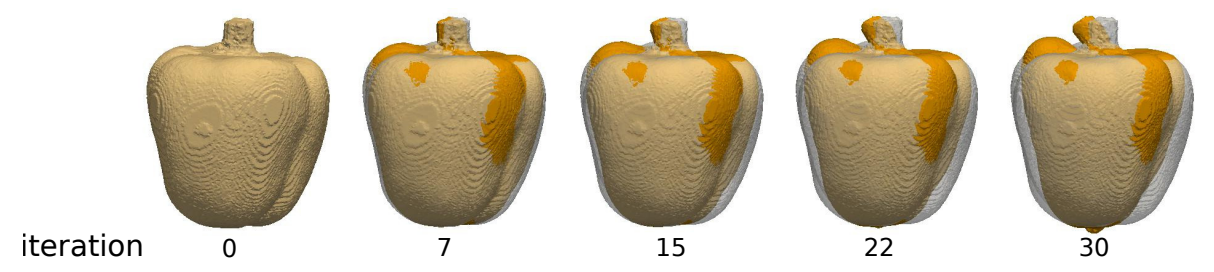

Fig. 5: Iterative shape deformation of a reference bell pepper towards a target bell pepper. For each iteration, the deformed reference mesh in orange and the original reference mesh is visualized in light gray.

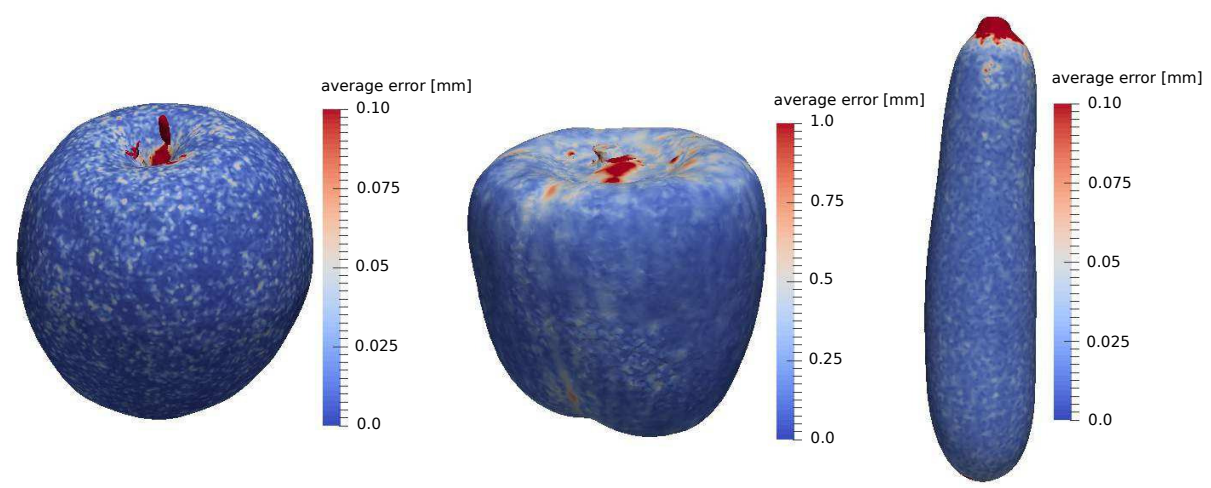

Fig. 6: Geometric error maps of the apple, bell pepper and zucchini. The errors were calculated for each instance in the population and averaged per object class. 


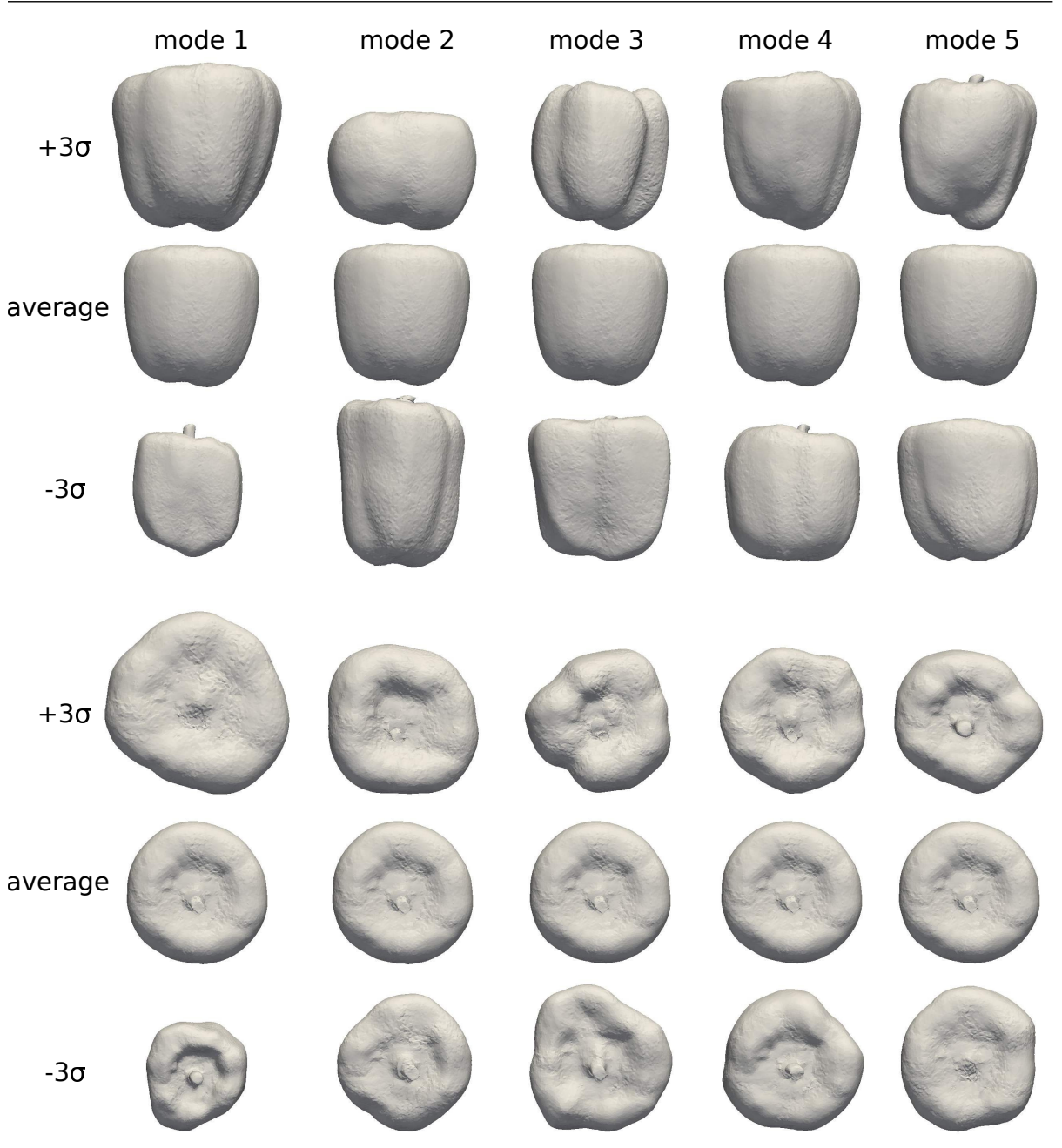

Fig. 7: A front view and a top view of the first five shape modes of the bell pepper shape model, plus and minus three standard deviations. 

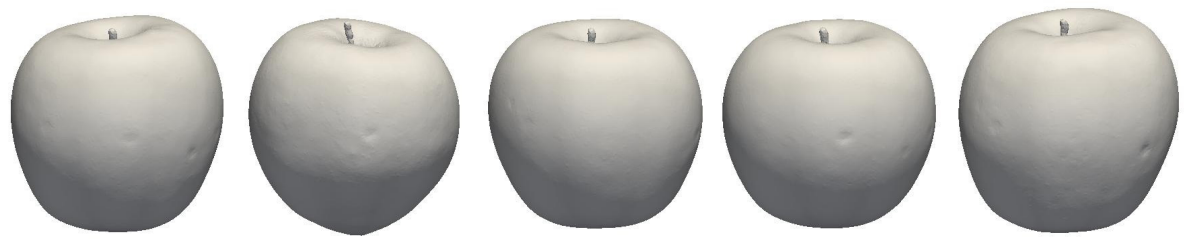

(a) Five randomly generated apple instances.
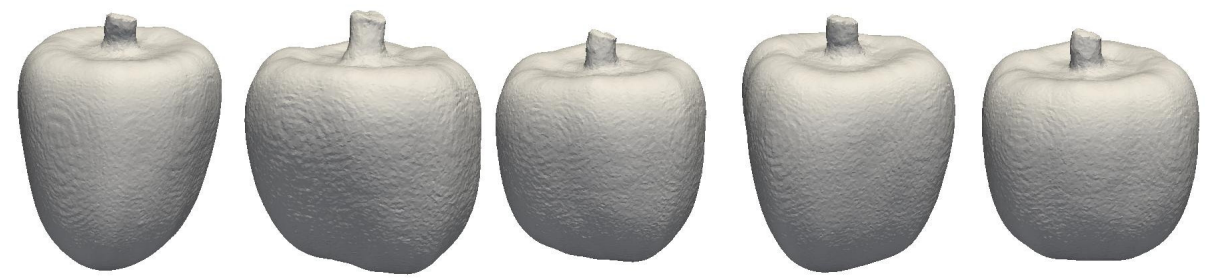

(b) Five randomly generated bell pepper instances.
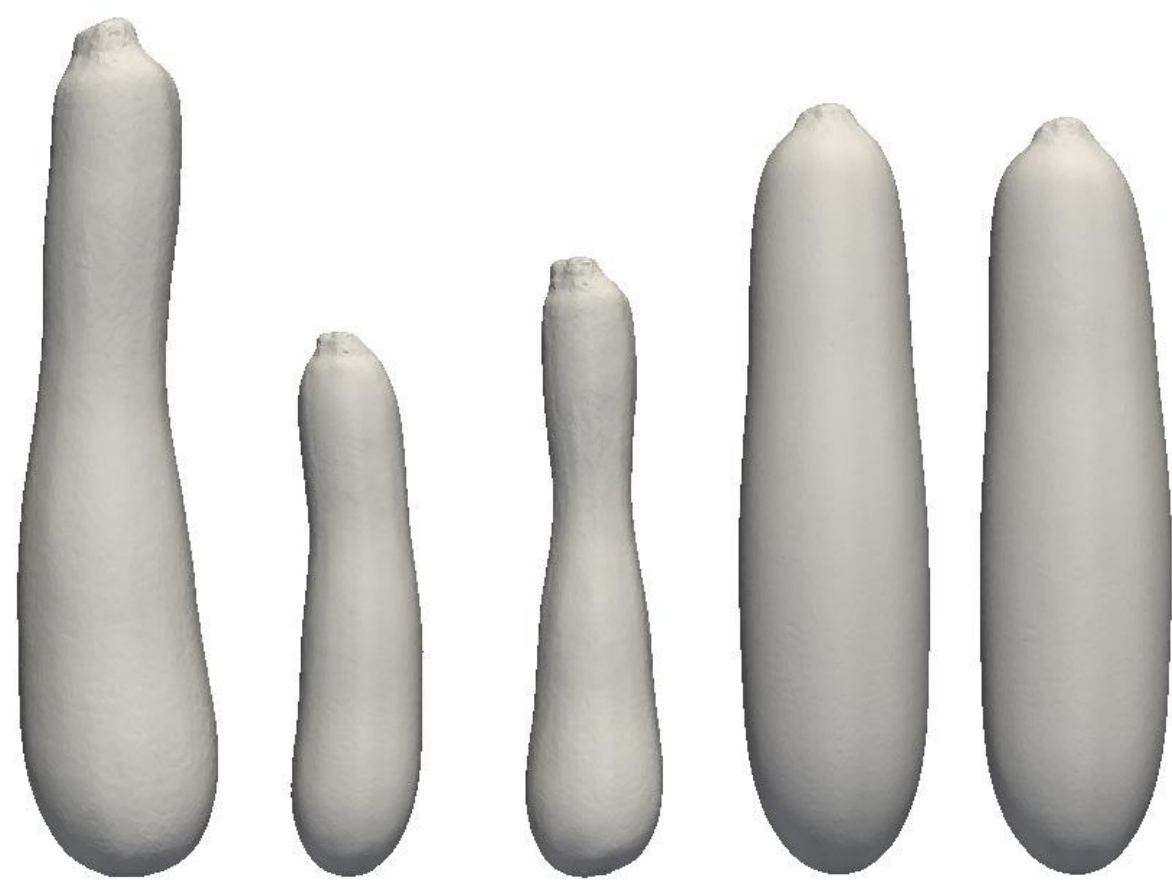

(c) Five randomly generated zucchini instances.

Fig. 8: Randomly generated horticultural products, created by applying a random weight vector to the first 5 eigenmodes of the shape model. 


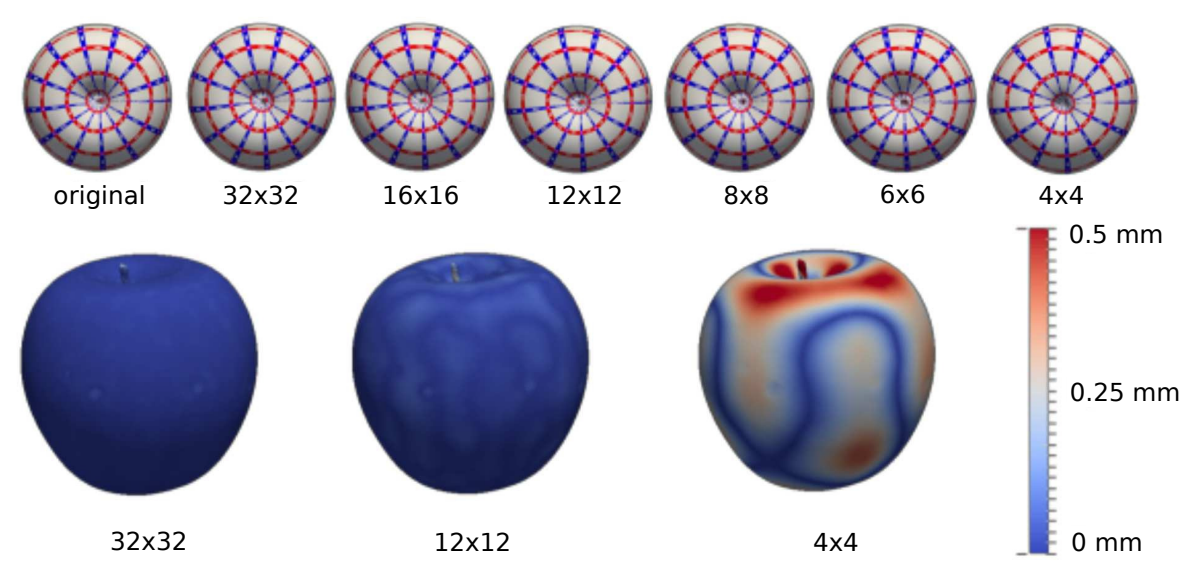

Fig. 9: Upper row: top view of parameterized apple shape model with isoparametric curves. B-spline approximations with different number of control points on the B-spline grid. Bottom row: distance, in $\mathrm{mm}$ between the original surface and the approximation.

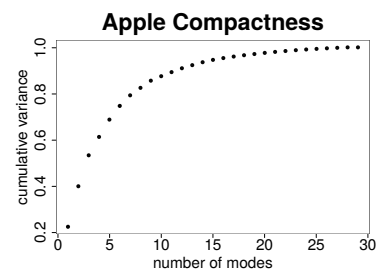

(a) Apple

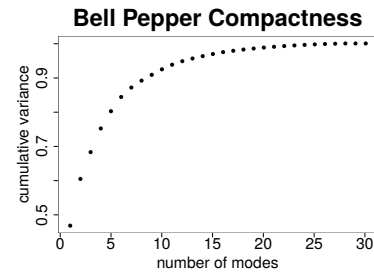

(b) Bell pepper

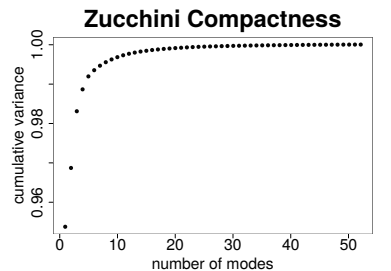

(c) Zucchini

Fig. 10: The compactness measure for the three types of horticultural products.

The cumulative variance was normalized for each model so that the total was $100 \%$. 


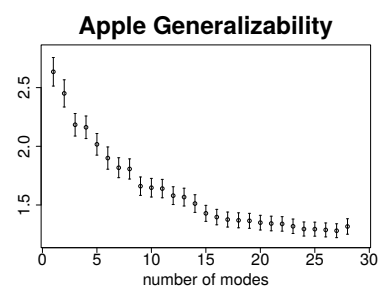

(a) Apple, $N_{t}=30$

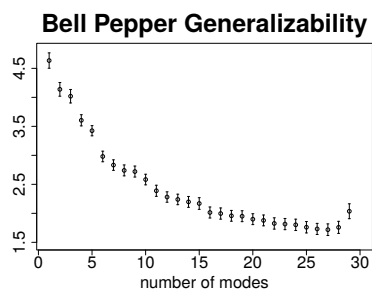

(b) Bell pepper, $N_{t}=30$

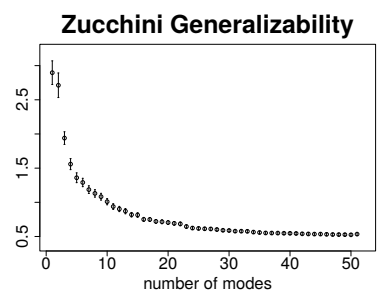

(c) Zucchini, $N_{t}=52$

Fig. 11: The generalizability measure for the three types of horticultural products, in $m m$ per vertex. The error flags represent the standard errors on the mean distance.

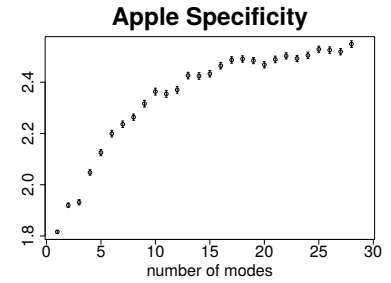

(a) Apple, $N_{t}=30$

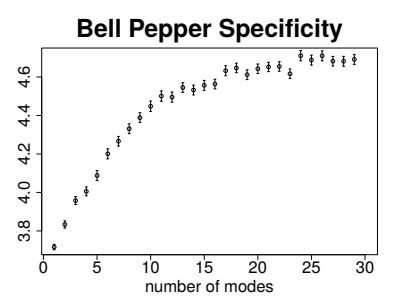

(b) Bell pepper, $N_{t}=30$

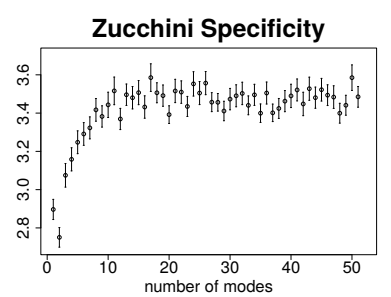

(c) Zucchini, $N_{t}=52$

Fig. 12: The specificity measure for the three types of horticultural products, in $m m$ per vertex. The error flags represent the standard errors on the mean distance. 


\begin{tabular}{c|c|c|c} 
& apple & bell pepper & zucchini \\
\hline width & $82.28 \pm 3.66$ & $69.30 \pm 11.41$ & $83.36 \pm 13.30$ \\
depth & $81.81 \pm 4.18$ & $68.07 \pm 11.81$ & $70.17 \pm 1.97$ \\
height & $84.50 \pm 4.74$ & $84.23 \pm 9.72$ & $284.04 \pm 38.71$
\end{tabular}

Table 1: Average and standard deviation of the dimensions of the fruit and vegetables in $m m$.

\begin{tabular}{c|c|c} 
apple & bell pepper & zucchini \\
\hline $0.015 \pm 0.011$ & $0.106 \pm 0.026$ & $0.027 \pm 0.007$
\end{tabular}

Table 2: Average and standard deviation of the distance between the deformed reference surface and target surface in $\mathrm{mm}$.

\begin{tabular}{c|c|c} 
stem & bottom & average \\
\hline $3.97 \pm 2.60$ & $4.84 \pm 3.15$ & $4.41 \pm 2.32$
\end{tabular}

Table 3: Average and standard deviation of the distance between the manually annotated markers and automatically derived markers from the shape model on 10 bell pepper instances in $m m$. 